Discrete Comput Geom 33:165-180 (2005)

DOI: $10.1007 / \mathrm{s} 00454-004-1101-y$

\title{
Finite Coverings in the Hyperbolic Plane*
}

\author{
Károly Böröczky, Jr. \\ Alfréd Rényi Institute of Mathematics, \\ P.O. Box 127, H-1364 Budapest, Hungary \\ carlos@renyi.hu
}

\begin{abstract}
We prove that if a (th $r$ )-convex domain in the hyperbolic plane is covered by $n \geq 2$ circular discs of radius $r$, then the density of the covering is larger than $2 \pi / \sqrt{27}$. The density bound is optimal, and the condition of (th $r$ )-convexity is essentially optimal. Combining our result with earlier estimates yields that if at least two non-overlapping equal circular discs cover a given circular disc in a surface of constant curvature, then the density of the covering is larger than $2 \pi / \sqrt{27}$.
\end{abstract}

\section{Introduction}

We call a compact subset $C$ of the Euclidean plane $E^{2}$, the sphere $S^{2}$ or the hyperbolic plane $H^{2}$ a convex domain if int $C \neq \emptyset$, and the segment connecting any two points of $C$ is contained in $C$. In the spherical case we assume that either $C=S^{2}$ or $C$ is contained in a closed hemisphere. The area of $C$ is denoted by $A(C)$. Given a covering of $C$ by $n$ equal circular discs, the density of the covering is the ratio of the total area of the $n$ circular discs to $A(C)$.

All the basic estimates for coverings of a convex domain $C$ by $n \geq 2$ equal circular discs in $E^{2}$ and on $S^{2}$ were verified in the 1940s and 1950s. In particular, L. Fejes Tóth [9], [10] proved that the density of such a covering is always larger than $2 \pi / \sqrt{27}$ if $C=S^{2}$, or $C$ is a convex domain in $E^{2}$ (some gaps in the proof in the Euclidean case were filled by G. Fejes Tóth [8]). Furthermore, Molnár [18] verified the same density bound for coverings of arbitrary spherical convex domains. Naturally $2 \pi / \sqrt{27}$ is the optimal lower bound in both spaces as hexagonal arrangements of small equal circular discs exhibit. On the other hand, such a general estimate does not hold for large $r$ in the hyperbolic plane; namely, for any $\varepsilon$ and $n$ there exist large $r$ and a covering of some

\footnotetext{
* This research was supported in part by OTKA Grants 43520 and 42769. This paper was written during a stay at Ohio State University, Columbus, Ohio, USA.
} 
convex domain by $n$ circular discs of radius $r$ such that the density is at most $1+\varepsilon$ (see Remark 1.3(ii)). Therefore it was not clear how to generalize the results in $E^{2}$ and on $S^{2}$ to the hyperbolic plane. In this paper we complete the picture; namely, we point out the right class of convex domains in $H^{2}$, and verify the density estimate $2 \pi / \sqrt{27}$ for finite coverings of these domains.

We recall that hyper-cycles of associated distance $r$ are the complete curves whose points are of distance $r$ from a given line on the one hand (see, say, [16]), and the complete curves of constant geodesic curvature th $r$ on the other hand (see, say, [14]). We say that a compact set $C$ is (th $r$ )-convex if it contains any arc of constant geodesic curvature th $r$ that connects two of its points. Equivalently, $C$ is a convex domain with the property that any boundary point $x$ of $C$ is contained in some hyper-cycle of associated distance $r$ such that $C$ lies on the convex side of the curve. Typical examples are circular discs, and the family of points whose distance from a given compact convex set is at most $r$.

Theorem 1.1. If $a$ (th $r$ )-convex domain $C$ in $H^{2}$ is covered by $n \geq 2$ circular discs of radius $r$, then the density of the covering is larger than $2 \pi / \sqrt{27}$.

Remark. It is equivalent to say that $A(C)<\sqrt{27}(\operatorname{ch} r-1) \cdot n$.

Since any circular disc is (th $r$ )-convex, Theorem 1.1 together with the results of $\mathrm{L}$. Fejes Tóth [9], [10] and Molnár [18] yield

Corollary 1.2. If at least two non-overlapping equal circular discs cover a given circular disc in $E^{2}, S^{2}$ or $H^{2}$, then the density of the arrangement is larger than $2 \pi / \sqrt{27}$.

We discuss the optimality of Theorem 1.1. The statements are verified at the end of the proof of Theorem 1.1.

\section{Remark 1.3.}

(i) The density bound $2 \pi / \sqrt{27}$ of Theorem 1.1 is optimal.

(ii) (th $r$ )-convexity is essentially the optimal condition in Theorem 1.1 for large $r$. More precisely, given any $\varepsilon \in(0,1)$, if $\varrho=r-\ln (6 / \varepsilon)$ for large $r$ and $n \geq 2$, then there exists a covering of density at most $1+\varepsilon$ of some (th $\varrho$ )-convex domain by $n$ circular discs of radius $r$.

(iii) While for given $r$ and $n$, the density bound $2 \pi / \sqrt{27}$ is readily not optimal, there exists a covering of some (th $r$ )-convex domain by $n$ circular discs of radius $r$ such that the density is less than $\pi / \eta=3.5644 \ldots$ where $\operatorname{sh} \eta=1$.

(iv) If $r \leq 0.6425$, then the density of a covering of any convex domain by $n \geq 2$ circular discs of radius $r$ is larger than $2 \pi / \sqrt{27}$ (no need for (th $r$ )-convexity).

In the case of packings of equal circular discs inside a convex domain, all the analogous results have been known for a long time. The optimal density bound $\pi / \sqrt{12}$ was verified by L. Fejes Tóth [9], [10] in the Euclidean plane and if the density of a spherical packing is measured with respect to $S^{2}$. For any spherical convex container, the bound $\pi / \sqrt{12}$ is due to Molnár [17]. Finally, the hyperbolic case was treated by Bezdek; namely, he 
showed the analogue of Corollary 1.2 in [1], and the analogue of Theorem 1.1 in [2]. We note that in case of packings of $n$ circular discs of radius $r$ inside (th $r$ )-convex domains, the density is at most $2 \pi / r$ for large $r$ (see [5]), hence it becomes arbitrary small as $r$ grows. This phenomenon is in contrast with the behaviour of coverings because there exits a covering of density less than four for any $r$ and $n$ (see Remark (ii)).

Next we discuss the so-called "triangle bound" for coverings in $E^{2}, S^{2}$ or $H^{2}$. We consider the regular triangle $T(r)$ of circumradius $r$ in $H^{2}$, and the three circular discs of radius $r$ centred at the vertices of the triangle. We define $\tau_{H^{2}}(r)$ to be the ratio of the sum of the areas of the three circular sectors lying in $T(r)$ to the area of $T(r)$. The corresponding spherical notion is denoted by $\tau_{S^{2}}(r)$, and the corresponding Euclidean ratio is $2 \pi / \sqrt{27}$. We consider a covering of a convex domain by $n \geq 2$ circular discs of radius $r$ in $E^{2}$ or in $S^{2}$ where $r<\pi / 2$ is assumed in the spherical case. Then the density of the covering is larger than $2 \pi / \sqrt{27}$ and $\tau_{S^{2}}(r)$, respectively. This was proved in the Euclidean plane and when the whole $S^{2}$ is covered by L. Fejes Tóth [10]. When a spherical convex domain different from $S^{2}$ is covered then we provide a quick proof in the Appendix (since $\tau_{S^{2}}(r)>2 \pi / \sqrt{27}$ according to L. Fejes Tóth [11, Section 37], it is a stronger estimate than the one of Molnár [18]). Concerning the hyperbolic case, it follows by $\tau_{H^{2}}(r)>2 \sqrt{3} / \pi$ (see Section 37 of [11]) and Remark 1.3(ii) that if $r$ is large, then the triangle bound does not hold for suitable coverings of certain convex domains. However, $\tau_{H^{2}}(r)<2 \pi / \sqrt{27}$ (see Section 37 of [11]) and Theorem 1.1 yields the triangle bound for for any $r$ if (th $r$ )-convex domains are covered.

Corollary 1.4. If a (th $r$ )-convex domain in $H^{2}$ is covered by $n \geq 2$ circular discs of radius $r$, then the density of the covering is larger than $\tau_{H^{2}}(r)$.

We note that the density of infinite coverings of the hyperbolic plane is an intricate notion. One cannot define it simply using elementary cells like the Delone cells or the Dirichlet-Voronoi cells (see [3]), but it is possible to define using ergodic theory for "most coverings" (see [6] and [7]). Since the triangle bound holds for every Delone cell (see [4]), the results of Bowen and Radin [6] show that the density of any infinite covering by circular discs of radius $r$ is at least $\tau_{H^{2}}(r)$ (whenever density exists), and equal to $\tau_{H^{2}}(r)$ if the covering is coming from a side to side tiling by regular triangles of circumradius $r$. Therefore we have a stronger bound for finite coverings of (th $r$ )-convex domains (see Theorem 1.1) than for infinite coverings. For further related results, see the surveys [12] and [15].

The paper is organized as follows: Section 2 constructs the corresponding Delone complex, and Section 3 verifies the key Lemma 3.1 concerning finite coverings. In Section 4 we estimate some quantities occurring in Lemma 3.1, and Theorem 1.1 is finally proved in Section 5. The Appendix discusses the triangle bound for finite coverings of spherical and Euclidean convex domains.

\section{The Delone Complex for Finite Coverings}

In this section we construct the Delone simplicial cell complex associated to a finite covering of some convex domain in $H^{2}$, and establish the main properties. 
Let a convex domain $C$ in $H^{2}$ be covered by $n \geq 2$ different circular discs of radius $r$ in a way that int $\mathrm{C}$ contains the centres $x_{1}, \ldots, x_{n}$ of the discs. We call a circular disc $B$ a supporting circular disc if its centre lies in $C$, its interior contains no $x_{i}$, and at least one of the following two possibilities hold: either $\partial B$ contains at least three $x_{i}$ 's, or the centre of $B$ lies in $\partial C$, and $\partial B$ contains at least two $x_{i}$ 's. To any supporting circular disc, we assign the convex hull of the $x_{i}$ 's in the disc. Since the common secant of two intersecting circular discs separates the associated convex hulls, we obtain a cell complex with vertices $x_{1}, \ldots, x_{n}$, which is called the Delone cell complex associated to the covering. Any triangulation of this cell complex using the same vertices is called a Delone simplicial cell complex.

For any edge $e$ of a simplicial complex $\Sigma$, a free side of $e$ (if it exists at all) is a half-plane bounded by the line of $e$ such that no triangle of $\Sigma$ containing $e$ lies in that half-plane. We write $b(\Sigma)$ to denote the total number of free sides of the edges.

Lemma 2.1. Let a convex domain $C$ in $H^{2}$ be covered be $n$ different circular discs of radius $r$ whose centres lie in int $\mathrm{C}$. If $\Sigma$ is an associated Delone simplicial complex $\Sigma$, then

(i) $\Sigma$ is connected;

(ii) $2 n=b(\Sigma)+f_{2}(\Sigma)+2$;

(iii) the at most $b(\Sigma)$ discs centred at the boundary of supp $\Sigma$ cover $\partial C$;

(iv) any edge and triangle of $\Sigma$ is of length at most $2 r$ and of area at most $A(T(r))$, respectively.

Proof. Let $\Sigma^{\prime}$ be the associated Delone cell complex, whose structure is easier to understand in terms of the Dirichlet-Voronoi complex $\Sigma_{\mathrm{DV}}$. Thus we write $D_{i}$ to denote the set of points in $C$ whose distance from $x_{i}$ is not larger than the distance from any other $x_{j}$, and define $\Sigma_{\mathrm{DV}}$ to be the cell decomposition of $C$ determined by $D_{1}, \ldots, D_{n}$. In particular, the Euler formula yields that $\chi\left(\Sigma_{\mathrm{DV}}\right)=1$.

Now the vertices of $\Sigma_{\mathrm{DV}}$ of degree at least three are the centres of the supporting circular discs. In addition, $x_{i_{1}} x_{i_{2}}$ is an edge of $\Sigma^{\prime}$ if and only if either of the following two possibilities occur: $D_{i_{1}}$ and $D_{i_{2}}$ share a common edge, or there exists a common endpoint of an edge of $\Sigma_{\mathrm{DV}}$ in $D_{i_{1}} \cap \partial C$ and an edge of $\Sigma_{\mathrm{DV}}$ in $D_{i_{2}} \cap \partial C$. We deduce that $\Sigma^{\prime}$ is connected, and $\chi\left(\Sigma^{\prime}\right)=\chi\left(\Sigma_{\mathrm{DV}}\right)=1$.

Turning to $\Sigma$, (i) and (iii) readily follow. Since $b(\Sigma)$ is the number of edges of $\Sigma$ on the boundary of supp $\Sigma$ where edges that are not contained in any of the cells are counted twice, (ii) is a consequence of $\chi(\Sigma)=1$. Finally we conclude (iv) as each supporting circular disc is of radius at most $r$.

\section{A Covering Inequality}

In this section a fundamental inequality for finite coverings of a convex domain by equal discs in $H^{2}$ is established. First we introduce some notions. Let $B(r)$ denote a circular disc of radius $r$ in $H^{2}$. We consider two secants of $B(r)$ that have a common endpoint and the same length, and are orthogonal to each other, and write $\Psi(r)$ to denote the cap 
cut off by one of these secants from $B(r)$. In order to handle coverings of (th $r$ )-convex sets, we introduce the following relatives of rectangles and squares: A hyper-rectangle is a convex domain, which is bounded by two segments of length $2 a$ that share a common perpendicular bisector $l$, and by the two hyper-cycle arcs whose points are of distance $a$ from $l$. In addition, we define $Q(r)$ to be a hyper-rectangle of maximal area contained in $B(r)$.

Lemma 3.1. For $r>0$, let the convex domain $C$ in $H^{2}$ be covered by $n \geq 2$ circular discs of radius $r$ in a way that each circular disc is needed to cover $C$, each centre is contained in int $C$, and no three of the $n$ centres lie on any circle centred at some point of $\partial C$. If $\Sigma$ is an associated Delone simplicial complex, then

(i) $A(C) \leq A(\operatorname{supp} \Sigma) \cdot \operatorname{ch} r+A(B(r))+b(\Sigma) / 2 \cdot(A(B(r))-4 A(\Psi(r))$;

(ii) $A(C) \leq A(\operatorname{supp} \Sigma) \cdot \operatorname{ch} r+A(B(r))+b(\Sigma) / 2 \cdot A(Q(r))$ if $C$ is (th $r)$-convex.

In course of the proof for Lemma 3.1, we need the following auxiliary statement:

Proposition 3.2. Let $B_{1}$ and $B_{2}$ be circular discs of radius $r$ with intersecting interiors in $H^{2}$, and let l be a line intersecting $B_{1} \cap B_{2}$ in a way that the centres of $B_{1}$ and $B_{2}$ lie on the same convex side of $l$. If $\Xi_{1}$ and $\Xi_{2}$ denote the part of $B_{1} \backslash B_{2}$ and $B_{2} \backslash B_{1}$, respectively, cut off by the other side of $l$, then

$$
A\left(\Xi_{1}\right)+A\left(\Xi_{2}\right)+A\left(B_{1} \cap B_{2}\right) \geq 4 A(\Psi(r)) .
$$

Analogously, if $l$ is a hyper-cycle of associated distance $r$, and the centres of $B_{1}$ and $B_{2}$ lie on the same convex side of $l$, then

$$
A\left(\Xi_{1}\right)+A\left(\Xi_{2}\right)+A\left(B_{1} \cap B_{2}\right) \geq A(B(r))-A(Q(r)) .
$$

Proof. Let $L=A\left(\Xi_{1}\right)+A\left(\Xi_{2}\right)+A\left(B_{1} \cap B_{2}\right)$, and let $l_{0}$ be the line of the common secant of $B_{1}$ and $B_{2}$. We may assume that $l$ passes through a common point $p$ of $\partial B_{1}$ and $\partial B_{2}$. Now the sum $A\left(\Xi_{1}\right)+A\left(\Xi_{2}\right)$ is minimal if $l \cap B_{1}$ and $l \cap B_{2}$ have the same length, hence we may also assume that $l$ is orthogonal to the line $l_{0}$.

If $l$ is a line, then $L$ is twice the sum of the areas of the two caps of $B_{1}$ cut off by $l$ and by $l_{0}$. Under the condition that $l$ and $l_{0}$ is orthogonal, the sum of the areas of the caps is minimal when the secants of $l$ and $l_{0}$ are of the same length, which in turn yields Proposition 3.2 in this case.

If $l$ is a hyper-cycle of associated distance $r$, then we may replace $l$ with the hypercycle $l^{\prime}$ that passes through $p$, and whose points are of distance $(d(p, q)) / 2$ from the perpendicular bisector of the common secant of $B_{1}$ and $B_{2}$. Then $L$ is the difference of the area of $B_{1}$ and the inscribed hyper-rectangle determined by $l^{\prime}, p$ and $q$, and hence Proposition 3.2 follows by the maximality of $A(Q(r))$.

Proof of Lemma 3.1. We present the proof of (i) in detail, and sketch the necessary changes for (ii) at the end. The family of points on $\partial C$ whose minimal distance from the vertices of $\Sigma$ is attained for two vertices of $\Sigma$ are in a bijective correspondence with the free sides of $\Sigma$. Thus their number is $b=b(\Sigma)$, and we write $x_{1}, \ldots, x_{b}$ to denote these 
points on $\partial C$ in clockwise order. We set $x_{0}=x_{b}$ and $x_{b+1}=x_{1}$. When speaking about the $\operatorname{arc} x_{i-1} x_{i}$, we mean the clockwise oriented arc from $x_{i-1}$ to $x_{i}$ on $\partial D$. We write $z_{i}$ and $z_{i+1}$ to denote the two vertices of $\Sigma$ closest to $x_{i}$, where we assume that the triangle $x_{i} z_{i+1} z_{i}$ is clockwise oriented for $i=1, \ldots, b$. We note that $z_{i}$ and $z_{j}$ may coincide for $i \neq j$. In addition, let $B_{i}$ be the circular disc in the covering whose centre is $z_{i}$.

First we consider the case $n=2$, and hence $b=2$. Then $\Sigma$ has no triangles, and only one edge $z_{1} z_{2}$. There exists a supporting line $l$ to $C$ that passes through $B_{1} \cap B_{2}$, and we deduce (i) by Proposition 3.2.

Thus let $n \geq 3$. We write $m_{i}$ to denote the midpoint of the segment $z_{i} z_{i+1}$, and assign to each $z_{i}$ the domain $\Pi_{i}$ bounded by the broken line $x_{i-1} m_{i-1} z_{i} m_{i} x_{i}$ and the $\operatorname{arc} x_{i-1} x_{i}$ on $\partial C$. Therefore,

$$
A(C)=A(\operatorname{supp} \Sigma)+\sum_{i=1}^{b} A\left(\Pi_{i}\right) .
$$

Next let $\widetilde{\Pi}_{i}$ be the sector of $B_{i}$, which contains $x_{i}$ and $x_{i-1}$, and is cut off by the two rays starting from $z_{i}$ and passing through $m_{i-1}$ and $m_{i}$, respectively. Since the total sum of the angles of $\Sigma$ is $f_{2}(\Sigma) \pi-A(\operatorname{supp} \Sigma)$, we deduce by Lemma 2.1(ii) that

$$
\begin{aligned}
\sum_{i=1}^{b} A\left(\widetilde{\Pi}_{i}\right) & =n \cdot A(B(r))-\frac{f_{2}(\Sigma) \pi-A(\operatorname{supp} \Sigma)}{2 \pi} \cdot A(B(r)) \\
& =\left(\frac{A(\operatorname{supp} \Sigma)}{2 \pi}+\frac{b+2}{2}\right) \cdot A(B(r)),
\end{aligned}
$$

which in turn yields by $A(B(r))=2 \pi(\operatorname{ch} r-1)$ that

$$
A(C)=A(\operatorname{supp} \Sigma) \cdot \operatorname{ch} r+\frac{b+2}{2} \cdot A(B(r))-\sum_{i=1}^{b} A\left(\tilde{\Pi}_{i} \backslash \Pi_{i}\right) .
$$

Let $\sigma_{i}$ denote the maximal open convex arc of $\partial C$ which lies in int $B_{i}$ and passes through $x_{i-1}$ and $x_{i}$. Now both $B_{i-1}$ and $B_{i+1}$ are needed to cover $C$, hence $\sigma_{i}$ connects a point of $B_{i-1} \cap \partial B_{i}$ with a point of $B_{i+1} \cap \partial B_{i}$, and cuts $B_{i}$ into two domains. We write $\Omega_{i}$ to denote the part not containing $z_{i}$, and claim that

$$
2 \sum_{i=1}^{b} A\left(\tilde{\Pi}_{i} \backslash \Pi_{i}\right)=\sum_{i=1}^{b} A\left(\Omega_{i} \backslash \Omega_{i+1}\right)+A\left(\Omega_{i+1} \backslash \Omega_{i}\right)+A\left(B_{i} \cap B_{i+1}\right) .
$$

Removing $\Pi_{i}$ from $\tilde{\Pi}_{i} \cap C$, we are left with two disjoint convex sets, and write $\Phi_{i}^{+}$and $\Phi_{i}^{-}$to denote the one cut off by the segment $x_{i} m_{i}$ and $x_{i-1} m_{i-1}$, respectively. In addition, we write $\Lambda_{i}^{+}$and $\Lambda_{i}^{-}$to denote the part of $\Omega_{i} \backslash \widetilde{\Pi}_{i}$ that is cut off by the half-line $z_{i} m_{i}$ and $z_{i} m_{i-1}$, respectively ( $\Lambda_{i}^{+}$or $\Lambda_{i}^{-}$might be the empty set). Then

$$
A\left(\tilde{\Pi}_{i} \backslash \Pi_{i}\right)=A\left(\Omega_{i}\right)+A\left(\Phi_{i}^{+}\right)-A\left(\Lambda_{i}^{+}\right)+A\left(\Phi_{i}^{-}\right)-A\left(\Lambda_{i}^{-}\right) .
$$

Since all $B_{i-1}, B_{i}$ and $B_{i+1}$ are needed to cover $C$, it is not hard to see that $\sigma_{i} \backslash \sigma_{i+1}$, $\sigma_{i} \cap \sigma_{i+1}$ and $\sigma_{i+1} \backslash \sigma_{i}$ are consecutive connected arcs in clockwise order along $\partial C$. In 
particular, one-half of $B_{i} \cap B_{i+1}$ cut off by the line $z_{i} z_{i+1}$ can be obtained from $\Omega_{i} \cap \Omega_{i+1}$ by removing $\Lambda_{i}^{+}$and $\Lambda_{i+1}^{-}$, and adding $\Phi_{i}^{+}$and $\Phi_{i+1}^{-}$. We deduce that

$$
\begin{gathered}
A\left(\Omega_{i}\right)+A\left(\Omega_{i+1}\right)+2 \cdot\left(A\left(\Phi_{i}^{+}\right)-A\left(\Lambda_{i}^{+}\right)+A\left(\Phi_{i+1}^{-}\right)-A\left(\Lambda_{i+1}^{-}\right)\right) \\
=A\left(\Omega_{i} \backslash \Omega_{i+1}\right)+A\left(\Omega_{i+1} \backslash \Omega_{i}\right)+A\left(B_{i} \cap B_{i+1}\right) .
\end{gathered}
$$

Therefore multiplying both sides of (3) by two, and summing the resulting formulae for $i=1, \ldots, b$ yields (2).

Let $l_{i}$ be a supporting line to $C$ at $x_{i}$. Then the part of $B_{i} \backslash B_{i+1}$ (of $B_{i+1} \backslash B_{i}$ ) cut off by $l_{i}$ is contained in $\Omega_{i} \backslash \Omega_{i+1}$ (in $\Omega_{i+1} \backslash \Omega_{i}$ ). We conclude via Proposition 3.2 that

$$
A(C) \leq A(\operatorname{supp} \Sigma) \cdot \operatorname{ch} r+\frac{b+2}{2} \cdot A(B(r))-\frac{b}{2} \cdot 4 A(\Psi(r)),
$$

which in turn yields Lemma 3.1(i). Finally if $C$ is (th $r$ )-convex, then the only change in the proof is that $l_{i}$ is a supporting hyper-cycle to $C$ of associated distance $r$.

We note that the idea of how to handle the circular discs covering the boundary of $C$ is due to L. Fejes Tóth [10].

\section{Preliminary Estimates}

In course of the proof Theorem 1.1, we frequently need inequalities of the form $f(x)>$ $g(x)$ for $x>0$ and for differentiable functions $f$ and $g$. If $f(0) \geq g(0)$, and $f^{\prime}(x)>$ $g^{\prime}(x)$ can be verified by rearrangement and possibly using simple estimates like $\operatorname{sh} x>x$, then we say that $f(x)>g(x)$ follows by derivation.

The proof Theorem 1.1 is based on Lemma 3.1(ii), therefore we need some estimates on the quantities occurring in that inequality. We start with $A(Q(r))$ :

Proposition 4.1. Let $r>0$. Then

(i) $A(Q(r))<4 \cdot(\operatorname{ch} r-1)$;

(ii) $A(Q(r))<\frac{8}{3} \cdot \operatorname{ch} r$;

(iii) $A(Q(r))<\frac{7}{3} \cdot \operatorname{ch} r$ if $\operatorname{ch} r \leq 3.5$.

Proof. For $0<a<r$, let $R_{a}$ be a hyper-rectangle inscribed into $B(r)$ such that the common perpendicular bisector $l$ of the two straight line sides intersects $R_{a}$ in a segment of length $2 a$. Then $A\left(R_{a}\right)=4 a \cdot \operatorname{sh} \varrho$ where $\varrho$ is the distance of the hypercycle arcs from $l$ (see p. 478 of [16]). Now the formula $\operatorname{ch} r=\operatorname{ch} \varrho \cdot \operatorname{ch} a$ yields that $d \varrho / d a=-(\operatorname{sh} a \cdot \operatorname{ch} \varrho) /(\operatorname{ch} a \cdot \operatorname{sh} \varrho)$ as $a$ varies. Therefore differentiating $a \cdot \operatorname{sh} \varrho$ with respect to $a$ shows that $A\left(R_{a}\right)$ is maximal if and only if

$$
\operatorname{ch}^{2} r=\frac{\operatorname{ch}^{3} a}{\operatorname{ch} a-a \cdot \operatorname{sh} a} .
$$

From now on, let $a$ satisfy (4), hence

$$
A(Q(r))=\frac{4 a^{3 / 2} \sqrt{\operatorname{sh} a}}{\sqrt{\operatorname{ch} a-a \cdot \operatorname{sh} a}} .
$$


It follows by derivation that $\operatorname{ch} a-a \cdot \operatorname{sh} a$ is monotone decreasing, and hence there exists a unique positive $a_{0}\left(a_{0}=1.1996 \ldots\right)$ satisfying

$$
\operatorname{ch} a_{0}-a_{0} \cdot \operatorname{sh} a_{0}=0 .
$$

In particular, $a<a_{0}$.

First we prove (i), which is equivalent to

$$
a^{3 / 2} \sqrt{\operatorname{sh} a}<\operatorname{ch}^{3 / 2} a-\sqrt{\operatorname{ch} a-a \cdot \operatorname{sh} a} .
$$

Now derivation yields that $\operatorname{ch} a-a \operatorname{sh} a$ is at most $1-(a / 2) \operatorname{sh} a$, and hence its square root is at most $1-(a / 4) \operatorname{sh} a$. Since $a^{3 / 2} \sqrt{\operatorname{sh} a}$ is at most $a \cdot \operatorname{sh} a$, it is sufficient to prove

$$
1+\frac{3}{4} a \cdot \operatorname{sh} a<\operatorname{ch}^{3 / 2} a .
$$

Squaring the derivatives of both sides leads to the inequality

$$
\operatorname{sh}^{2} a+a^{2} \operatorname{ch}^{2} a+2 a \operatorname{ch} a \cdot \operatorname{sh} a<4 \operatorname{ch} a \cdot \operatorname{sh}^{2} a .
$$

This inequality follows by using $\operatorname{ch}^{2} a=1+\operatorname{sh}^{2} a$ on the left-hand side, and $4 \operatorname{ch} a>$ $2 \operatorname{ch} a+2+a^{2}$ on the right-hand side. In turn, we conclude (i).

We turn to the other two estimates for $A(Q(r))$. We note that

$$
A(Q(r))=4 q(a) \cdot \operatorname{ch} r \quad \text { for } \quad q(a)=\left(\frac{a}{\operatorname{ch} a}\right)^{3 / 2} \cdot \sqrt{\operatorname{sh} a} .
$$

Here derivation yields that $a / \operatorname{ch} a$ is monotone increasing for $a \leq a_{0}$, thus $q(a)$ is monotone increasing, as well. We define $a_{1}$ by the property that ch $r=3.5$ corresponds to $a=a_{1}$. We deduce by numerical calculations that $q\left(a_{0}\right)<\frac{8}{3}$ and $q\left(a_{1}\right)<\frac{7}{3}$, which in turn yield (ii) and (iii).

In order to estimate expression $A(\operatorname{supp} \Sigma)$ that occurs in Lemma 3.1, we use two bounds. The first is concerned with $T(r)$.

Proposition 4.2. Let $r>0$. Then

(i) $A(T(r))<6 \sqrt{3} \cdot((\operatorname{ch} r-1) /(3 \operatorname{ch} r+1))$;

(ii) $A(T(r)) \cdot \operatorname{ch} r<\pi \cdot(\operatorname{ch} r-1)$;

(iii) $A(T(r)) \cdot \operatorname{ch} r<3.025 \cdot(\operatorname{ch} r-1)$ if $\operatorname{ch} r \leq 3.5$.

Proof. We note that

$$
A(T(r))=\pi-6 \cdot \arctan \frac{1}{\sqrt{3} \operatorname{ch} r}=6 \cdot \arctan \frac{\sqrt{3}(\operatorname{ch} r-1)}{3 \operatorname{ch} r+1} .
$$

Therefore the inequality $\arctan t<t$ for $t>0$ yields (i).

In order to verify (ii) and (iii), we introduce the variable $t=1 / \sqrt{3} \mathrm{ch} r$. Since (ii) is equivalent with $\arctan t>((\pi \sqrt{3}) / 6) t$ for $0<t<1 / \sqrt{3}$, the strict concavity of $\arctan t$ yields (ii). Similarly, (iii) is equivalent with $\arctan t>(3.025 \pi / 6) t+(\pi-3.025) / 6$ for $1 /(3.5 \sqrt{3}) \leq t<1 / \sqrt{3}$, which holds again by the strict concavity of $\arctan t$ and by numerical calculations. 
For $A(\operatorname{supp} \Sigma)$, we also need another type of bound. Since each of the $b=b(\Sigma)$ free sides of $\Sigma$ is of length at most $2 r$, we deduce by the isoperimetric inequality for polygons (see [13]) that $A(\operatorname{supp} \Sigma)$ is at most the area of the regular $b$-gon of side length $2 r$; namely,

$$
A(\operatorname{supp} \Sigma) \leq 2 b \cdot\left(\arccos \frac{\cos (\pi / b)}{\operatorname{ch} r}-\frac{\pi}{b}\right)
$$

Proposition 4.3. Let $0<c_{1}<1$ and $c_{2}>0$. Then for $b \geq 3$,

$$
\arccos \left(c_{1} \cdot \cos \frac{\pi}{b}\right)-\frac{c_{2}}{b} \leq \max \left\{\arccos c_{1}, \arccos \frac{c_{1}}{2}-\frac{c_{2}}{3}\right\} .
$$

Proof. The function $f(b)=\arccos c_{1} \cdot \cos (\pi / b)-c_{2} / b$ of $b$ satisfies

$$
f^{\prime}(b)=\frac{1}{b^{2}} \cdot\left(c_{2}-c_{1} \pi \cdot\left(\frac{1-c_{1}^{2}}{\sin ^{2}(\pi / b)}+1\right)^{-1 / 2}\right) .
$$

Now the expression in the parentheses is monotone increasing; therefore $f(b)$ is either decreasing, or increasing, or decreasing up to some $b_{0}$ and increasing after $b_{0}$. Thus $f(b)$ is at most the maximum of $f(3)$ and $\lim _{b \rightarrow \infty} f(b)$.

\section{Proof of Theorem 1.1}

If $C$ can be covered by one circular disc of radius $r$, then we are done, thus we assume that it is not the case. If the centre $x$ of one of the circular discs lies outside of $C$, then we may replace this disc by the circular disc of radius $r$ whose centre is the closest point of $C$ to $x$. Therefore we may assume that

the centre of each of the $n$ circular discs is contained in $C$.

First we prove a slightly weaker version of Theorem 1.1; namely, we allow the density of the covering to be $2 \pi / \sqrt{27}$. Possibly increasing the common radius $r$ and varying the centres, we may assume that each centre is contained in int $C$, no proper subset of the $n$ discs cover $C$, and no three of the $n$ centres lie on any circle centred at some point of $\partial C$. Now we may apply Lemma 3.1. Let $\Sigma$ be an associated Delone simplicial complex, and we simply write $f_{2}$ and $b$ instead of $f_{2}(\Sigma)$ and $b(\Sigma)$, respectively. Then $\Sigma$ has $n$ vertices, and Lemma 2.1 yields that $n=\frac{1}{2} f_{2}+\frac{1}{2} b+1$. According to Lemma 3.1(ii), it is sufficient to prove

$$
\begin{aligned}
A(\operatorname{supp} \Sigma) \cdot & \operatorname{ch} r+2 \pi(\operatorname{ch} r-1)+\frac{1}{2} b \cdot A(Q(r)) \\
& <3 \sqrt{3}\left(\frac{1}{2} f_{2}+\frac{1}{2} b+1\right) \cdot(\operatorname{ch} r-1) .
\end{aligned}
$$


If $n=2$, then $b=2, f_{2}=0$ and $A(\Sigma)=0$. In particular, we deduce by Proposition 4.1(i) that (9) is a consequence of

$$
2 \pi \cdot(\operatorname{ch} r-1)+4 \cdot(\operatorname{ch} r-1)<2 \cdot 3 \sqrt{3} \cdot(\operatorname{ch} r-1),
$$

which inequality readily holds by numerical calculation. Therefore let

$$
n \geq 3, \quad \text { and hence } \quad b \geq 3
$$

follows from the connectivity of supp $\Sigma$ (see Lemma 2.1).

Case I: $\operatorname{ch} r \geq 3.5$. According to Proposition 4.1(i) and Proposition 4.2(ii), (9) is the consequence of the inequality

$$
\pi f_{2}(\operatorname{ch} r-1)+2 \pi(\operatorname{ch} r-1)+2 b(\operatorname{ch} r-1) \leq \frac{3 \sqrt{3}}{2}\left(b+f_{2}+2\right) \cdot(\operatorname{ch} r-1)
$$

thus (9) holds if $f_{2} \leq(3 \sqrt{3}-4) /(2 \pi-3 \sqrt{3}) \cdot b-2$.

Therefore let $f_{2} \geq(3 \sqrt{3}-4) /(2 \pi-3 \sqrt{3}) \cdot b-2$. Now we apply Proposition 4.1(ii) and (7), and hence (9) is the consequence of the inequality

$$
\begin{aligned}
2 b(\arccos & \left.\frac{\cos (\pi / b)}{\operatorname{ch} r}-\frac{\pi}{b}\right) \cdot \operatorname{ch} r+2 \pi(\operatorname{ch} r-1)+\frac{4}{3} b \operatorname{ch} r \\
< & \frac{3 \sqrt{3}}{2} \cdot\left(b+f_{2}+2\right) \cdot(\operatorname{ch} r-1) .
\end{aligned}
$$

We deduce by $f_{2} \geq(3 \sqrt{3}-4) /(2 \pi-3 \sqrt{3}) \cdot b-2$, by dividing through (10) by $2 b \cdot \operatorname{ch} r$ and by regrouping the terms that it is sufficient to verify

$$
\arccos \frac{\cos (\pi / b)}{\operatorname{ch} r}-\frac{\pi}{\operatorname{ch} r} \cdot \frac{1}{b}<\frac{3 \sqrt{3} \cdot(\pi-2)}{2 \cdot(2 \pi-3 \sqrt{3})} \cdot\left(1-\frac{1}{\operatorname{ch} r}\right)-\frac{2}{3} .
$$

According to Proposition 4.3, this estimate follows from inequalities

$$
\begin{array}{r}
\arccos \frac{1}{\operatorname{ch} r}+\frac{3 \sqrt{3}(\pi-2)}{2(2 \pi-3 \sqrt{3})} \cdot \frac{1}{\operatorname{ch} r}<\frac{3 \sqrt{3}(\pi-2)}{2(2 \pi-3 \sqrt{3})}-\frac{2}{3} \\
\arccos \frac{1}{2 \operatorname{ch} r}-\frac{\pi}{3} \cdot \frac{1}{\operatorname{ch} r}+\frac{3 \sqrt{3}(\pi-2)}{2(2 \pi-3 \sqrt{3})} \cdot \frac{1}{\operatorname{ch} r}<\frac{3 \sqrt{3}(\pi-2)}{2(2 \pi-3 \sqrt{3})}-\frac{2}{3} .
\end{array}
$$

We introduce the variable $s=1 / \mathrm{ch} r$. Since the derivative of both

$$
\arccos s+\frac{3 \sqrt{3} \cdot(\pi-2)}{2 \cdot(2 \pi-3 \sqrt{3})} \cdot s \text { and } \arccos \frac{s}{2}+\left(\frac{3 \sqrt{3} \cdot(\pi-2)}{2 \cdot(2 \pi-3 \sqrt{3})}-\frac{\pi}{3}\right) \cdot s
$$

are positive for $0<s \leq 1 / 3.5$, and both inequalities (12) and (13) hold for $\operatorname{ch} r=3.5$, we conclude (9). 
Case II: $2.5 \leq \mathrm{ch} r \leq 3.5$. We handle this case very similarly to Case I. We deduce by Proposition 4.1(i) and Proposition 4.2(iii) that (9) is the consequence of the inequality

$$
\begin{gathered}
3.025 f_{2} \cdot(\operatorname{ch} r-1)+2 \pi \cdot(\operatorname{ch} r-1)+2 b \cdot(\operatorname{ch} r-1) \\
\leq \frac{3 \sqrt{3}}{2} \cdot\left(b+f_{2}+2\right) \cdot(\operatorname{ch} r-1),
\end{gathered}
$$

thus (9) holds if $f_{2} \leq(3 \sqrt{3}-4) /(6.05-3 \sqrt{3}) \cdot b-(4 \pi-6 \sqrt{3}) /(6.05-3 \sqrt{3})$.

Therefore let $f_{2} \geq(3 \sqrt{3}-4) /(6.05-3 \sqrt{3}) \cdot b-(4 \pi-6 \sqrt{3}) /(6.05-3 \sqrt{3})$. Now we apply Proposition 4.1(iii) and (7), and hence (9) is the consequence of the inequality

$$
\begin{aligned}
2 b \cdot(\arccos & \left.\frac{\cos (\pi / b)}{\operatorname{ch} r}-\frac{\pi}{b}\right) \cdot \operatorname{ch} r+2 \pi \cdot(\operatorname{ch} r-1)+\frac{7}{6} b \cdot \operatorname{ch} r \\
< & \frac{3 \sqrt{3}}{2} \cdot\left(b+f_{2}+2\right) \cdot(\operatorname{ch} r-1) .
\end{aligned}
$$

We deduce by the condition on $f_{2}$, by dividing through (14) by $2 b \cdot \operatorname{ch} r$, and by regrouping the terms that it is sufficient to verify

$$
\begin{gathered}
\arccos \frac{\cos (\pi / b)}{\operatorname{ch} r}-\left(\frac{\pi}{\operatorname{ch} r}+\frac{3 \sqrt{3}}{4} \cdot\left(2-\frac{4 \pi-6 \sqrt{3}}{6.05-3 \sqrt{3}}\right) \cdot\left(1-\frac{1}{\operatorname{ch} r}\right)\right) \cdot \frac{1}{b} \\
<\frac{3 \sqrt{3}}{4} \cdot\left(1+\frac{3 \sqrt{3}-4}{6.05-3 \sqrt{3}}\right) \cdot\left(1-\frac{1}{\operatorname{ch} r}\right)-\frac{7}{12} .
\end{gathered}
$$

Now this inequality can be proved similarly to (11) in Case I: first one eliminates $b$ using Proposition 4.3, then verifies the two resulting inequalities by introducing the new variable $s=1 / \mathrm{ch} r$ where $0<s \leq 1 / 2.5$.

Case III: $\operatorname{ch} r \leq 2.5$. We deduce by the bounds $f_{2} \geq A(\operatorname{supp} \Sigma) / A(T(r))$ and Proposition 4.1(i) that (9) is the consequence of the inequality

$$
\begin{aligned}
A(\operatorname{supp} \Sigma) & \cdot \operatorname{ch} r+2 \pi(\operatorname{ch} r-1)+2 b(\operatorname{ch} r-1) \\
& <\frac{3 \sqrt{3}}{2} \cdot\left(b+\frac{A(\operatorname{supp} \Sigma)}{A(T(r))}+2\right) \cdot(\operatorname{ch} r-1),
\end{aligned}
$$

which can be rewritten in the form

$$
\begin{aligned}
A(\operatorname{supp} \Sigma) & \cdot\left(\operatorname{ch} r-\frac{3 \sqrt{3}(\operatorname{ch} r-1)}{2 A(T(r))}\right)+(2 \pi-3 \sqrt{3}) \cdot(\operatorname{ch} r-1) \\
& <b \cdot\left(\frac{3 \sqrt{3}}{2}-2\right) \cdot(\operatorname{ch} r-1) .
\end{aligned}
$$

Proposition 4.2(i) yields that the coefficient of $A(\operatorname{supp} \Sigma)$ is at most $\frac{1}{4}(\operatorname{ch} r-1)$, and we apply (7) to $A(\operatorname{supp} \Sigma)$. Therefore after dividing by $(b / 2)(\operatorname{ch} r-1),(16)$ will follow if the inequality

$$
\arccos \frac{\cos (\pi / b)}{\operatorname{ch} r}-\frac{6 \sqrt{3}-3 \pi}{b}<3 \sqrt{3}-4
$$


is verified. Now $b$ can be eliminated using Proposition 4.3 as above, and the two resulting inequalities are the consequences of $1 / \operatorname{ch} r \geq 1 / 2.5$. We conclude (9), and in turn Theorem 1.1 up to the possibility that the density equals $2 \pi / \sqrt{27}$.

Finally we prove the strict inequality. We fix an interval $[p, q]$ such that $2.5,3.5 \notin$ ( $p, q$ ]. The proof of (9) yields a positive constant $c$ depending on $p, q, n, f_{2}(\Sigma)$ and $b(\Sigma)$ such that if $r \in[p, q]$, then the right-hand side of (9) minus the left-hand side is at least $c$. Given $n$, we have only finitely many choices for $f_{2}(\Sigma)$ and $b(\Sigma)$, hence we may assume that $c$ depends only on $p, q, n$. Let $r \in[p, q)$. It follows by (8) that for any $r<r^{\prime}<q$, there exists a covering of $C$ by $n$ circular discs of radius $r^{\prime}$ such that each centre lies in the interior of $C$. Since the density of any such covering is at least $2 \pi / \sqrt{27}+c_{0}$ for some positive $c_{0}$ depending on $p, q$ and $n$, we conclude the strict inequality in Theorem 1.1.

Proof of the Statements of Remark 1.3. Statement (i) simply follows from the observation that if $r$ is small, then a circular disc of radius $\sqrt{r}$ can be covered by circular discs of radius $r$ in a way such that the density is close to $2 \pi / 3 \sqrt{3}$.

The proofs of (ii) and (iii) use the quadrilateral $L(\varrho)$, which has three right angles, and the acute angle is enclosed by two sides of length $\varrho$.

In order to verify (ii) for given $0<\varepsilon<1$, we define $\varrho=r-\ln (6 / \varepsilon)$ for large $r$. Let the quadrilateral $p_{1} p_{2} p_{3} p_{4}$ satisfy the following properties: $p_{1}$ is the centre of $B(r)$, and $p_{3} \in \partial B(r)$. In addition, the opposite angles at $p_{2}$ and $p_{4}$ are right angles, the side $p_{1} p_{4}$ is of length $\varrho$, and the angle at $p_{1}$ is the acute angle of $L(\varrho)$. We write $p_{3}^{\prime}$ to denote the second intersection point of $\partial B(r)$ and the hyper-cycle of associated distance $\varrho$ that is orthogonal to the segment $p_{3} p_{2}$ at $p_{3}$, and $p_{1}$ lies on the convex side. We claim that if $r$ is large, then

$$
\frac{\angle p_{3}^{\prime} p_{1} p_{2}}{2 \pi}<\frac{\varepsilon}{8}
$$

Let $\alpha(r)$ and $\beta(r)$ denote the angles $\angle p_{3} p_{1} p_{4}$ and $\angle p_{3} p_{1} p_{2}$, hence $\angle p_{3}^{\prime} p_{1} p_{2}=2 \alpha(r)+$ $\beta(r)$. In order to simplify the formulae, we write $\lambda=6 / \varepsilon$. We deduce by the Laws of Sine and Cosine that

$$
\tan \alpha(r)=\left(\frac{\operatorname{sh}^{2} r}{\operatorname{sh}^{2} \varrho}-1\right)^{-1 / 2} \sim \frac{1}{\sqrt{\lambda^{2}-1}}
$$

on the one hand, and

$$
\tan \beta(r) \sim \frac{1}{\sqrt{2} \lambda-\sqrt{\lambda^{2}-1}}<\frac{\sqrt{2}+1}{\sqrt{\lambda^{2}-1}}
$$

on the other hand. Since $\sqrt{\lambda^{2}-1}>\sqrt{35} / \varepsilon$, we conclude the claim (18).

Now let $a^{*}$ denote the length of the side $p_{2} p_{3}$, hence $\varrho<a^{*}<r$. We place $n \geq 2$ circular discs of radius $r$ in a way that the centres are aligned, and the common secants of consecutive discs are of length $2 a^{*}$. For each secant, we draw the two hyper-cycles of associated distance $\varrho$, which are orthogonal to the secant at the endpoints, and whose convex sides contain the secant. We define $C$ to be the family of points of the union of the $n$ discs that lie on the convex side of each of the $2(n-1)$ hyper-cycles, and note 
that $C$ is a (th $\varrho$ )-convex domain. For any of the given discs and for a corresponding secant, (18) yields a circular sector of angle $\varepsilon \pi / 2$ that contains the parts of the disc cut off by the secant and the two orthogonal hyper-cycles (assuming that $r$ is large enough). Therefore the density of the covering is less than $1+\varepsilon$.

Turning to (iii), we may assume that $n \geq 4$. Let $k=\lfloor(n-2) / 2\rfloor$, and let $a(r)$ be the length of the shorter sides of $L(r)$, which satisfies sh $a(r)=$ th $r$. Given a segment of length $2 k \tilde{a}(r)$, we define the (th $r$ )-convex domain $C$ to be the family of points whose distance from the segment is at most $r$. Then $C$ can be dissected into two semi-circular discs of radius $r$, and $2 k$ congruent copies of the following convex domain $\Pi(r): \Pi(r)$ is bounded by three segments and an arc of some hyper-cycle of associated distance $r$ where the middle segment is of length $2 \tilde{a}(r)$, and the other two segments are orthogonal to it, and are of length $r$. If $\operatorname{ch} r \geq(\sqrt{5}+1) / 2$, then the shorter diagonal of $L(r)$ is of length at most $r$, hence $C$ can be covered by $n$ circular discs of radius $r$, and the density of the covering is at most the maximum of 3 and $A(B(r)) / A(\Pi(r))$. Now derivation shows that $A(B(r)) / A(\Pi(r))=\pi(\operatorname{ch} r-1) /(a(r) \operatorname{sh} r)$ is increasing if $\operatorname{ch} r \geq(\sqrt{5}+1) / 2$, hence it is less than its limit $\pi /(\operatorname{arcsh} 1)$ at infinity. Finally, it is not hard to construct coverings of suitably small density if $\operatorname{ch} r \leq(\sqrt{5}+1) / 2$.

Let $r \leq 0.6425$. We prove (iv) similarly to the proof of Theorem 1.1 in the case ch $r \leq 2.5$, only using Lemma 3.1(i) instead of Lemma 3.1(ii) for the covering of some convex domain by $n$ circular discs of radius $r$. We define $\psi(r)$ by the relation

$$
A(B(r))-4 A(\Psi(r))=\psi(r) \cdot(\operatorname{ch} r-1),
$$

hence $\psi(r)=(8 \operatorname{ch} r) /(\operatorname{ch} r-1) \cdot \arctan ((\operatorname{ch} r-1) /(\operatorname{ch} r+1))$. Introducing the variable $t=(\operatorname{ch} r-1) /(\operatorname{ch} r+1)$, it follows by derivation that $\psi(r)$ is increasing.

We replace the term $2 b(\operatorname{ch} r-1)$ by $(\psi(r) b / 2)(\operatorname{ch} r-1)$ on the left-hand side of (15), and it is sufficient to prove the resulting inequality. Therefore we replace the right-hand side of (17) by $3 \sqrt{3}-\psi(r)$. According to Proposition 4.3, the density of the covering is at least $2 \pi / 3 \sqrt{3}$ if

$$
\arccos \frac{1}{2 \operatorname{ch} r}-(2 \sqrt{3}-\pi) \leq 3 \sqrt{3}-\psi(r),
$$

which inequality holds for $r \leq 0.6425 \ldots$ by numerical calculations.

\section{Appendix}

First we prove the triangle bound for spherical coverings.

Theorem A.1. If $n \geq 2$ circular discs of radius $r$ on $S^{2}$ cover a spherical convex domain $C \neq S^{2}$, then the density of the covering is larger than $\tau_{S^{2}}(r)$.

Proof. Let $0<r<\pi / 2$. We define $B(r), T(r)$ and $\Psi(r)$ similarly to the hyperbolic case, and associate a Delone complex to any finite covering of a spherical convex domain $C$ that is different from $S^{2}$. In addition, we obtain the following analogue of Lemma 3.1(i): For $0<r<\pi / 2$, let the spherical convex domain $C \neq S^{2}$ be covered by $n \geq 2$ circular 
discs of radius $r$ in such a way that each circular disc is needed in order to cover $C$, each centre is contained in int $C$, and no three centres lie on a circle centred at a point of $\partial D$. If $\Sigma$ is an associated Delone simplicial complex, then

$$
A(C) \leq A(\operatorname{supp} \Sigma) \cdot \cos r+A(B(r))+\frac{b(\Sigma)}{2} \cdot(A(B(r))-4 A(\Psi(r))) .
$$

In turn, (19) leads to the following more general estimate: if a spherical convex domain $C \neq S^{2}$ is covered by $n \geq 2$ circular discs of radius $r$, then

$$
\begin{aligned}
A(C) \leq & \left(n-\frac{b}{2}-1\right) \cdot 2 \cos r \cdot A(T(r))+A(B(r)) \\
& +\frac{b}{2} \cdot(A(B(r))-4 A(\Psi(r))),
\end{aligned}
$$

where some $b$ discs of radius $r$ cover $\partial D$. Therefore Theorem A. 1 is the consequence of the inequality

$2 A(T(r)) \cos r \cdot\left(n-\frac{b}{2}-1\right)+A(B(r))+(A(B(r))-4 A(\Psi(r))) \cdot \frac{b}{2}<\frac{A(B(r))}{\tau_{S^{2}}(r)} \cdot n$.

We may assume that $b \geq 2$, hence it is sufficient to verify the estimates

$$
\begin{aligned}
2 A(T(r)) \cos r & <\frac{A(B(r))}{\tau_{S^{2}}(r)}, \\
A(B(r))+(A(B(r))-4 A(\Psi(r))) & <2 \cdot \frac{A(B(r))}{\tau_{S^{2}}(r)} .
\end{aligned}
$$

We define $\pi / 6<\alpha(r)<\pi / 2$ by $\cos r=1 /(\sqrt{3} \tan \alpha(r))$, and hence $A(T(r))=$ $6 \alpha(r)-\pi$ and $A(B(r)) / \tau_{S^{2}}(r)=2 \pi(6 \alpha(r)-\pi) / 6 \alpha(r)$. Therefore (21) can be written in the form

$$
\tan \alpha(r)-\frac{6}{\sqrt{3} \pi} \cdot \alpha(r)>0 .
$$

Since here equality holds for $\alpha=\pi / 6$, and the left-hand side is increasing for $\alpha \geq \pi / 6$, we conclude (21).

In order to verify (22), we define $\pi / 4<\beta(r)<\pi / 2$ by $\cos r=1 / \tan \beta(r)$. Then $A(B(r))=2 \pi \cdot(1-\cos r)$ yields that

$$
\Psi(r)=2 \beta(r) \cdot(1-\cos r)-\left(2 \beta(r)-\frac{\pi}{2}\right)=\frac{\pi}{2}-2 \beta(r) \cdot \cos r .
$$

In particular, (22) is equivalent with the inequality

$$
\frac{\pi}{3 \alpha(r)} \cdot \frac{1}{\cos r}+\frac{4}{\pi} \cdot \beta(r)<\frac{1}{\cos r}+2 .
$$

If $\alpha(r) \geq \pi / 3$, then (23) readily holds by $\beta(r)<\pi / 2$. Thus we assume that $\alpha(r) \leq \pi / 3$, and hence $x=1 / \cos r$ satisfies that $1 \leq x \leq 3$. We rewrite (23) in the form

$$
\frac{\pi}{3 \arctan (x / \sqrt{3})} \cdot\left(x-\frac{6}{\pi} \cdot \arctan \frac{x}{\sqrt{3}}\right)<x-\frac{4}{\pi} \cdot \arctan x .
$$


Here $x-(6 / \pi) \cdot \arctan (x / \sqrt{3}) \geq 0$ holds for $x \geq 1$, as can be checked by derivation. For $\lambda>0$, we define the auxiliary function

$$
f_{\lambda}(x)=\lambda \cdot\left(x-\frac{6}{\pi} \cdot \arctan \frac{x}{\sqrt{3}}\right)-\left(x-\frac{4}{\pi} \cdot \arctan x\right),
$$

whose second derivative with respect to $x$ is

$$
f_{\lambda}^{\prime \prime}(x)=\frac{2 x}{\pi \cdot\left(3+x^{2}\right)^{2}} \cdot\left(\frac{3 \sqrt{3} \cdot \lambda}{2}-\left(1+\frac{2}{1+x^{2}}\right)^{2}\right) .
$$

We verify (24) by dividing the interval $[1,3]$ into four subintervals, thus let the interval $\left[x_{1}, x_{2}\right]$ be either $[1,1.1],[1.1,1.4],[1.4,2.3]$ or $[2.3,3]$. We define $\lambda=\pi /(3 \cdot \arctan$ $\left.\left(x_{1} / \sqrt{3}\right)\right)$. Now numerical evaluation shows that $f_{\lambda}^{\prime \prime}\left(x_{1}\right)>0$, and hence $f_{\lambda}^{\prime \prime}(x)>0$ holds for $x \geq x_{1}$. Since $f_{\lambda}\left(x_{1}\right)$ and $f_{\lambda}\left(x_{2}\right)$ are negative by numerical evaluation, the convexity of $f_{\lambda}$ yields (24) for $\left[x_{1}, x_{2}\right]$. In turn, we conclude Theorem A.1.

Finally we remark that the Euclidean version of (20) says that if a convex domain $C$ in $E^{2}$ is covered by $n \geq 2$ unit discs, then

$$
A(C) \leq \frac{3 \sqrt{3}}{2} \cdot n-\left(\frac{3 \sqrt{3}}{4}-1\right) \cdot b+\pi-\frac{3 \sqrt{3}}{2},
$$

where some $b$ unit discs cover $\partial C$. This fundamental inequality is due to L. Fejes Tóth [10] and G. Fejes Tóth [8], therefore it could be properly named the Fejes Tóth inequality. If $b=1$, then $A(C) \leq \pi<(3 \sqrt{3} / 2) \cdot n$. Moreover, if $b \geq 2$, then (25) yields that $A(C)<(3 \sqrt{3} / 2) \cdot n$. This is the way L. Fejes Tóth [10] obtained the triangle bound for finite coverings of convex domains in $E^{2}$ by unit discs:

Theorem A.2 (L. Fejes Tóth). If a convex domain $C$ in $E^{2}$ is covered by $n \geq 2$ unit discs, then

$$
A(C)<\frac{3 \sqrt{3}}{2} \cdot n
$$

\section{Acknowledgement}

I thank a referee whose comments greatly improved the paper.

\section{References}

1. K. Bezdek, Ausfüllungen eines Kreises durch kongruente Kreise in der hyperbolische Ebene. Studia Sci. Math. Hungar., 17 (1982), 353-366.

2. K. Bezdek, Ausfüllungen in der hyperbolische Ebene durch endliche Anzahl kongruente Kreise. Ann. Univ. Sci. Budapest, 27 (1984), 113-124. 
3. K. Böröczky, Sphere packings in spaces of constant curvature, I (in Hungarian). Mat. Lapok, 25 (1974), 265-306.

4. K. Böröczky, The density of hyperbolic circle packings with respect to Delone triangles. Manuscript.

5. K. Böröczky, Jr., Discrete point sets in the hyperbolic plane. Studia Sci. Acad. Hungar., 39 (2002), 21-36.

6. L. Bowen, Ch. Radin, Densest packing of equal spheres in hyperbolic space, Discrete Comput. Geom., 29 (2003), 23-29.

7. L. Bowen, Ch. Radin, Optimally dense packings of hyperbolic space. Geom. Dedicata, accepted.

8. G. Fejes Tóth, Finite coverings by translates of centrally symmetric convex domains. Discrete Comput. Geom., 2 (1987), 353-363.

9. L. Fejes Tóth, Extremal distributions of points in the plane, on the surface of the sphere and in space. Univ. Francisco-Josephina. Kolozsvár. Acta Sci. Math. Nat., No. 23 (1944).

10. L. Fejes Tóth, Über dichteste Kreislagerung und dünnste Kreisüberdeckung. Comment. Math. Helv., 23 (1949), 342-349.

11. L. Fejes Tóth, Regular Figures. Pergamon Press, Oxford, 1964.

12. G. Fejes Tóth, W. Kuperberg, Packing and covering. In: Handbook of Convex Geometry, P.M. Gruber, J.M. Wills (eds.), North-Holland, Amsterdam, 1993, pp. 799-860.

13. A. Florian, Extremum problems for convex discs and polyhedra. In: Handbook of Convex Geometry, P.M. Gruber, J.M. Wills (eds.), North-Holland, Amsterdam, 1993, pp. 177-221.

14. E. Gallego, A. Reventós, Asymptotic behaviour of $\lambda$-convex sets in the hyperbolic plane. Geom. Dedicata, 76 (1999), 275-289.

15. P. Gritzmann, J.M. Wills, Finite packing and covering. In: Handbook of Convex Geometry, P.M. Gruber, J.M. Wills (eds.), North-Holland, Amsterdam, 1993, pp. 861-897.

16. G.E. Martin, The Foundations of Geometry and the Non-Euclidean Plane. Springer-Verlag, New York, 1996.

17. J. Molnár, Ausfüllung und Überdeckung eines konvexen sphärischen Gebieten durch Kreise, I. Publ. Math. Debrecen, 2 (1952), 266-275.

18. J. Molnár, Ausfüllung und Überdeckung eines konvexen sphärischen Gebieten durch Kreise, II. Publ. Math. Debrecen, 3 (1953), 150-157.

Received January 2, 2003, and in revised form January 15, 2004. Online publication June 18, 2004. 